\title{
Viabilidad y tasa de preñez de embriones producidos in vitro a partir de semen sexado comparado con semen convencional en Bos taurus y Bos indicus
}

\author{
Viability and pregnancy rate of embryos produced in vitro from sexed semen \\ compared to conventional semen in Bos taurus and Bos indicus
}

\author{
Lilian Bonilla León ${ }^{1,4}$, Andrés Mejía Gallego², Ramón Gómez Domínguez ${ }^{3}$, Martha \\ Torres Londoño ${ }^{3}$, Fabian Uribe García ${ }^{1}$
}

\section{RESUMEN}

\begin{abstract}
El estudio se realizó con la producción de embriones in vitro del laboratorio In Vitro Colombia ${ }^{\circledR}$ de 2015 . Se analizó la tasa de división embrionaria, la producción de embriones in vitro (PIV) y la tasa de preñez teniendo en cuenta el tipo de semen utilizado (sexado y convencional) para la fertilización y la especie bovina (Bos taurus, Bos indicus). Se analizaron datos de 840 sesiones de aspiración folicular, resultando 127503 oocitos puestos en cultivo in vitro provenientes de 5257 hembras. La tasa de división embrionaria para el semen sexado fue de $69.6 \%$ y para el semen convencional de $74.5 \%(p<0.001)$. Se obtuvo $20.8 \%$ de PIV para el semen sexado y de $26.5 \%$ para el convencional $(\mathrm{p}<0.001)$. El semen sexado de $B$. taurus se comportó mejor en términos de división embrionaria que el de $B$. indicus $(71.1$ vs. $64.4 \%)(\mathrm{p}<0.001)$, mientras que el semen convencional de $B$. indicus se comportó mejor que el de B. taurus $(76.0$ vs. $70.3 \%)(\mathrm{p}<0.001)$. En la PIV, el semen sexado de $B$. indicus se comportó mejor que el semen de $B$. taurus $(21.5$ y $20.7 \%$, respectivamente; $\mathrm{p}=0.008)$, mientras que con semen convencional la PIV de $B$. indicus fue de $28.2 \%$ frente a $22.2 \%$ de $B$. taurus $(\mathrm{p}<0.001$ ). No hubo diferencias significativas para las tasas de preñez. Se concluye que el uso de semen sexado para la producción de embriones bovinos in vitro es viable.
\end{abstract}

Palabras clave: aspiración folicular; OPU; transferencia de embriones; semen sexado; producción de embriones in vitro

\footnotetext{
${ }^{1}$ Facultad de Medicina Veterinaria y Zootecnia, Universidad del Tolima, Ibagué, Tolima, Colombia

${ }^{2}$ Facultad de Medicina Veterinaria y Zootecnia, Universidad Cooperativa de Colombia, sede Salado, Ibagué, Tolima, Colombia

${ }^{3}$ Laboratorio de Fecundación In Vitro, InVitro, Colombia

${ }^{4}$ E-mail: lory0101@yahoo.com

Recibido: 9 de febrero de 2018

Aceptado para publicación: 6 de agosto de 2018
} 
The study was carried out with the in vitro embryo production of the In Vitro Colombia ${ }^{\circledR}$ laboratory of 2015 . The embryo cleavage rate, the in vitro embryo production (IVP) and the pregnancy rate were analysed based on the type of semen used (sexed and conventional) for fertilization and on the bovine species (Bos taurus, Bos indicus). Data from 840 sessions of follicular aspiration were analysed, resulting in 127503 oocytes placed in in vitro culture from 5257 females. The embryonic cleavage rate for sexed semen was $69.6 \%$ and for conventional semen $74.5 \%(\mathrm{p}<0.001)$. It was obtained $20.8 \%$ of IVP for sexed semen and $26.5 \%$ for conventional semen $(\mathrm{p}<0.001)$. The sexed semen of $B$. taurus performed better in terms of embryonic cleavage than that of $B$. indicus (71.1 and 64.4\%, respectively $(\mathrm{p}<0.001)$, whereas the conventional semen of $B$. indicus performed better than $B$. taurus ( 76.0 and $70.3 \%$, respectively, $\mathrm{p}<0.001)$ In the IVP the sexed semen of $B$. indicus performed better than the semen of $B$. taurus (21.5 and $20.7 \%$, respectively, $\mathrm{p}=0.008$ ), whereas with conventional semen the IVP of $B$. indicus was $28.2 \%$ compared to $22.2 \%$ of $B$. taurus $(\mathrm{p}<0.001)$. There were no significant differences for pregnancy rates. It is concluded that the use of sexed semen to produce in vitro bovine embryos is viable.

Key words: ovum pick-up; OPU; embryo transfer; sexed semen; in vitro embryo production

\section{INTRODUCCIÓN}

El ciclo reproductivo natural en el bovino conduce al nacimiento de una cría por año en el mejor de los casos. Para mejorar la eficiencia reproductiva de animales de alto mérito genético se utilizan técnicas de reproducción asistida (Plourde et al., 2012). A mediados de la década del 80, la única tecnología de embriones que se aplicaba en la práctica para la multiplicación de la hembra bovina era la superovulación y transferencia de embriones (Multiple Ovulation and Embryo Transfer - MOET). Pronto se hizo evidente que esta técnica tenía límites y que podían desarrollarse otras técnicas para la producción de embriones requeridos por la industria ganadera. Con el tiempo se consideró la idea de aprovechar la cantidad de oocitos presentes en los ovarios (Galli y Lazzari, 2008). De esta forma, la fecundación in vitro (FIV) surgió como una alternativa a la superovulación y se ha convertido en la técnica de elección para la producción de embriones bovinos, especialmente en razas de Bos indicus (Viana et al., 2012).
Las tecnologías de reproducción asistida en animales de granja han sido utilizadas con éxito en todo el mundo, aumentan la importancia de las hembras en programas de mejoramiento genético y desempeñan un papel importante en la mejora de la precisión e intensidad de la selección de rasgos cuantitativos en vacas lecheras (Guerreiro et al., 2014). En condiciones normales de reproducción, la probabilidad de obtener hembra o macho es de $50 \%$, pero en las fincas ganaderas de producción de leche se busca tener mayores nacimientos de hembras, pues los machos tienen un retorno monetario mínimo para el productor (Barceló-Fimbres et al., 2011; Jo et al., 2014).

Es posible y económicamente viable la combinación del uso de semen sexado y la fecundación in vitro, ya que predeterminando el sexo del embrión se logrará una mayor eficiencia, especialmente en ganadería de leche (Barceló-Fimbres et al., 2011) al obtenerse más frecuencia de hembras (Rasmussen et al., 2013). Hinde et al. (2014) encontró, además, que el promedio de producción de lactancia era más alto en la se- 
gunda lactancia cuando las vacas gestaban una cría hembra.

El objetivo de este trabajo fue presentar datos de producción de embriones in vitro a gran escala, basado en el trabajo de campo y el laboratorio de In Vitro Colombia ${ }^{\circledR}$, con el fin de analizar la eficiencia del semen sexado en la producción de embriones in vitro y por medio de los promedios de producción comparar los resultados entre Bos indicus y Bos taurus sobre división embrionaria, producción de embriones y tasa de preñez.

\section{Materiales y Métodos}

Los datos fueron tomados por el laboratorio In Vitro Colombia ${ }^{\circledR}$ durante 2015, en varias zonas del país, donde se realizaron 840 sesiones de aspiración folicular, de las cuales se obtuvieron 127503 oocitos puestos en cultivo de 5257 hembras bovinas de diferente condición racial y categoría reproductiva. Las donadoras fueron seleccionadas según el interés del productor en cuanto a características productivas fenotípicas y genéticas intrínsecas de cada explotación. La edad de los animales fue variable.

Las variables objetivo de comparación fueron las tasas de división embrionaria, la producción de embriones y la tasa de preñez. La información se filtró de acuerdo a las variables a comparar (especie [Bos indicus, Bos taurus], tipo de semen [convencional, sexado]) y para el caso de la preñez se utilizaron los datos de embriones transferidos que tuvieron resultado de diagnóstico de gestación.

\section{Donadoras}

Las donantes de oocitos fueron animales libres de enfermedades reproductivas transmisibles, en condición corporal aceptable (mínimo 3, en escala de 1-5). Los animales seleccionados no estaban gestantes o tenían máximo 120 días de gestación al mo- mento de la aspiración folicular. Las donantes en este estudio se sometieron a la aspiración folicular sin sincronización previa de la onda folicular.

\section{Semen}

El semen que se utilizó en los procesos de fecundación in vitro provino de casas comerciales nacionales e internacionales y poseen entre 2 y 10 millones de espermatozoides en el caso de semen sexado y entre 10 y 40 millones de espermatozoides en el caso de semen convencional. La calidad y concentración del semen fue evaluada en el laboratorio por el técnico encargado de la fecundación in vitro (datos no presentados en este trabajo).

Se trabajó con hembras bovinas Bos indicus (Brahman, Guzerat, Gyr, Nelore) y Bos taurus (Simmental, Holstein, Angus, Blonde Dáquitania, Piemontese, Ayrsrhire, Charolais, Braunvieh, Jersey, Limousin, Normando, Pardo Suizo, Rojo Alemán, Wagyu).

\section{Aspiración Folicular (OPU)}

La aspiración folicular se llevó a cabo según la técnica descrita por Seneda et al. (2001). Los complejos de oocitos-cúmulos fueron clasificados de la siguiente forma: Grado 1) más de tres capas de material compacto de células del cúmulo; Grado 2) al menos una capa de células del cúmulo; Grado 3) desnudo; Grado 4) atrésico, con células cúmulos oscuras y signos de degeneración citoplasmática (Seneda et al., 2001). Los oocitos atrésicos se descartan y los demás (Grados 1, 2 y 3) se utilizan en el proceso de producción de embriones in vitro (PIV).

\section{Fertilización y Producción de Embriones in vitro}

Los oocitos recuperados fueron sometidos al tratamiento propuesto por Pontes et al. (2011). El material aspirado se filtró inmediatamente a través de un filtro EmCon con solución salina tamponada con fosfato 
(PBS-Nutricell, Brasil) suplementado con suero fetal bovino (SFB) al 5\%. Antes de la maduración in vitro (MIV), los complejos del cúmulo del oocito (COC) se lavaron tres veces con TCM-199 HEPES (Gibco Life Technologies, EEUU) suplementado con SFB al $10 \%$ y sulfato de gentamicina $50 \mathrm{~g}$ y una vez en bicarbonato TCM-199 (Gibco Life Technologies) (Pontes et al., 2011) suplementado con SFB al 10\%, $5 \mathrm{~g}$ de hormona luteinizante (LH, Ayerst, EEUU), $0.5 \mathrm{~g}$ hormona folículo estimulante (FSH - Folltropin, Vetrepharm, Canadá), $1 \mathrm{~g}$ de estradiol (17ß estradiol, Sigma E-8875), $2.2 \mathrm{~g}$ de piruvato (Sigma P-4562) y $50 \mathrm{~g}$ de gentamicina/ml de medio.

Los COC de cada categoría se cultivaron por separado para $24 \mathrm{~h}$ en $100 \mathrm{~L}$ gotas de medio de maduración bajo Petróleo mineral (D'Altomare, Brasil) en $39^{\circ} \mathrm{C}$ con $\mathrm{CO}_{2}$ al 5\% (Gordon, 1994; Smith et al., 1996). Para la FIV, dos pajillas se descongelaron durante 20 segundos en baño de maría a $35^{\circ} \mathrm{C}$. El semen fue lavado y centrifugado a través de un gradiente $90-45 \%$ de Percoll a $200 \mathrm{~g}$ durante $30 \mathrm{~min}$. El esperma fue capacitado usando heparina $(30 \mathrm{~g} / \mathrm{ml})$ y la motilidad fue estimulada por la adición de $40 \mu \mathrm{l} / \mathrm{ml}$ de factores de motilidad espermática y pemicilamida (PHE) (Bavister, 1989). La concentración de esperma se ajustó a $25 \times 10^{6}$ espermatozoides vivos/ml, y cada gota de fertilización recibió $4 \mu 1$ de esperma (concentración final 100×10 células por gota) (Seneda et al., 2001).

Después de la maduración, los COC se lavaron tres veces en el medio de pre-fertilización de TCM-199 suplementado con HEPES $25 \mathrm{mM}$ y albúmina sérica bovina (BSA) al 0.3\% (Sigma A-9647) y una vez en medio de fertilización TALP suplementado con $10 \mathrm{~g} / \mathrm{ml}$ de heparina y $160 \mu 1$ de solución PHE (Parrish et al., 1986, 1988). Después de la FIV, los oocitos y los COC se transfirieron a gotas de $100 \mu \mathrm{l}$ de medio de cultivo para embriones, un fluido sintético modificado del oviducto, SOFaa BSA, conteniendo $8 \mathrm{mg} / \mathrm{ml}$ de BSA (Sigma, EEUU) libre de ácido graso y glutamina $1 \mathrm{mM}$. Permanecieron en este medio entre 5 y 8 días. Este paso se realizó bajo condiciones de misma temperatura y ambiente gaseoso. La osmolaridad se mantuvo en $270-280 \mathrm{mOsmol} \mathrm{y} \mathrm{el} \mathrm{pH}$ fue de 7.4.

El semen para la FIV se separó en Percoll 90 al 45\% y se centrifugó (200 g) durante $30 \mathrm{~min}$, antes de ajustarse para obtener una concentración final de $100 \times 10^{3}$ espermatozoides viables por gota. La FIV se llevó a cabo durante $18 \mathrm{~h}$ a $39^{\circ} \mathrm{C}$ en una atmósfera de $\mathrm{CO}_{2}$ al $5 \%$ en aire. Después de la fecundación, las estructuras se lavaron y se transfirieron a $100 \mu \mathrm{l}$ de medio fluidizado de oviducto sintético modificado y se cubrieron con aceite mineral. El medio de cultivo se renovó para cada micropozo después de 3 y 5 días (alimentación), y después de 6 días fue llevado a la granja para transferir los embriones a las vacas receptoras (Perez et al., 2016).

Los embriones se clasificaron de acuerdo con los criterios IETS (Wright, 1998). Solo se consideraron embriones de grado I o II para transferir (embriones de mórula y blastocistos de 6 y 7.5 días de desarrollo). La calidad embrionaria, el número de células embrionarias de la masa celular interna y trofoectodermo, incidencia de apoptosis, capacidad de eclosión, anormalidades cromosómicas y expresión de genes específicos, han sido aceptados extensamente como determinantes de la calidad embrionaria (Sugimura et al., 2017).

\section{Sincronización de Receptoras}

La evaluación de la receptora para establecer su estado reproductivo y sanidad se hizo con ayuda de un equipo de ultrasonido (Mindray DP 50 y transductor lineal de 7.5 $\mathrm{MHz}$ ). Los animales seleccionados se sometieron a un protocolo de sincronización de estro hormonal, usando un dispositivo de progesterona intravaginal de liberación lenta de $0.5 \mathrm{~g}$ (Calier-Syntex) por 7 u 8 días, benzoato de estradiol de $1 \mathrm{mg} / \mathrm{ml}$ (CalierSyntex), cloprostenol $0.075 \mathrm{mg} / \mathrm{ml} \mathrm{y}$ gonadotropina coriónica equina Novormon (Calier-Syntex) (ver Figura 1). 


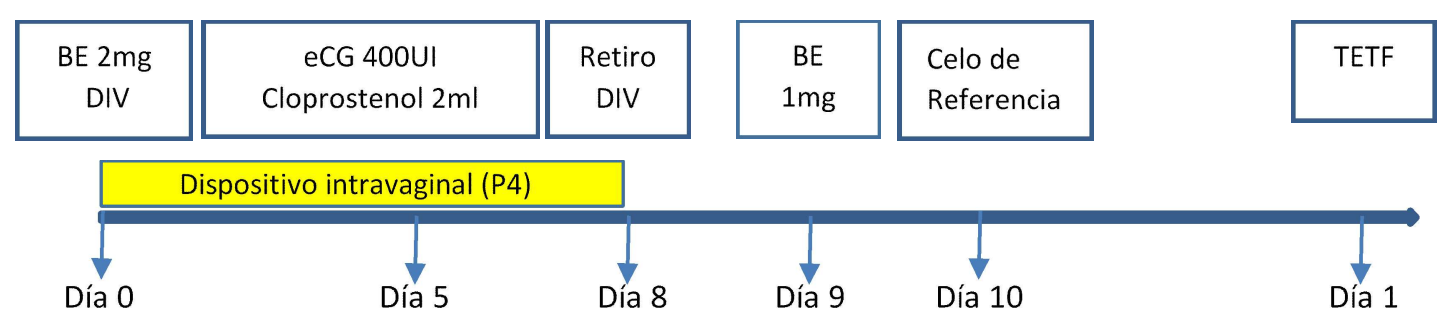

Figura 1. Protocolo de sincronización para transferencia de embriones a tiempo fijo (TETF)

\section{Transferencia de Embriones}

La transferencia de embriones se realizó a los 17 días de haber iniciado el protocolo de sincronización (Nasser, 2004), previa detección de un cuerpo lúteo mayor a $16 \mathrm{~mm}$ mediante ultrasonografía transrectal (Mindray DP 50 y transductor lineal de $7.5 \mathrm{MHz}$ ).

Para la transferencia, se aplicó anestesia epidural (4 $\mathrm{ml}$ de lidocaína) a la receptora, se limpió la zona perivulvar y el embrión fue colocado en la parte más distal del cuerno uterino ipsilateral al cuerpo lúteo resultante del protocolo. Los embriones de mórula y blastocistos de 6 a 7.5 d posfecundación fueron transferidos individualmente a cada receptora (Pontes et al., 2011).

\section{Diagnóstico de Gestación}

El diagnóstico de gestación se hizo a los 35-60 días de la transferencia de embriones, mediante ecografía sobre el útero de la receptora. Se identificó la presencia de vesícula gestacional y se corroboró la fetocardia positiva utilizando un ultrasonido modo $\mathrm{B}$ Mindray DP 10 con transductor de $7.5 \mathrm{mHz}$.

\section{Evaluación Estadística}

Se evaluó la tasa de clivaje o división embrionaria, la producción de embriones y la tasa de preñez según el tipo de semen utilizado y entre $B$. taurus vs. B. indicus.

Los datos obtenidos de los procedimientos de aspiración folicular (oocitos que entran a la maduración in vitro), de la división embrionaria y la tasa de preñez se analizaron utilizando el programa estadístico Minitab $16^{\circledR}$. En el caso de las variables de tipo categórico se realizó una comparación de proporciones: número de oocitos puestos a cultivo con la división embrionaria o clivaje y los embriones obtenidos. En el caso de la tasa de preñez se comparó la cantidad de embriones transferidos con los datos de preñez. El programa Minitab $16^{\circledR}$ aplica la prueba de Fisher para establecer si hay diferencia entre las proporciones.

\section{Resultados y Discusión}

Se entiende como principio que el desarrollo embrionario depende completamente de la integridad y del criterio de selección del oocito para la fertilización (Morotti et al., 2014). En general, la producción de embriones y la tasa de división embrionaria en el semen convencional se comportó mejor que el semen sexado, teniendo valores significativamente más altos.

Inaba et al. (2016) señalan que no obtuvieron diferencia significativa comparando los tipos de semen en la división de embriones ni en la producción de embriones in vitro. Por otro lado, Barceló-Fimbres et al. (2011) plantean que las diferencias obtenidas por el tipo de semen en la división embrionaria pudieron deberse a la cinética de penetración, que puede estar afectada por el procedimiento de sexaje del semen. Así mismo, Lonergan y Fair (2008) creen que un factor fundamental para este parámetro tiene que ver con la ca- 
Cuadro 1. Comparación de los parámetros de producción de embriones (\%) obtenidos del análisis de la producción in vitro, comparando semen de Bos Taurus y Bos indicus, $y$ entre el tipo de semen utilizado

\begin{tabular}{|c|c|c|c|c|c|c|c|c|c|}
\hline & \multicolumn{3}{|c|}{ Bos taurus } & \multicolumn{3}{|c|}{ Bos indicus } & \multicolumn{3}{|c|}{ Total } \\
\hline & 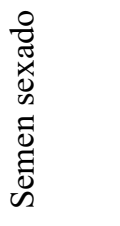 & 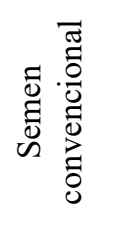 & $\mathrm{P}$ & 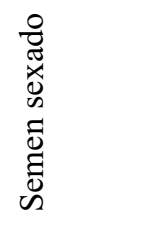 & 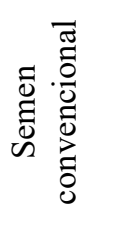 & $\mathrm{P}$ & 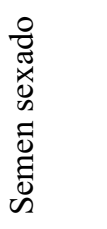 & 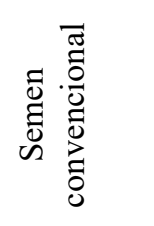 & $\mathrm{P}$ \\
\hline $\begin{array}{l}\text { División } \\
\text { embrionaria }\end{array}$ & $\begin{array}{c}71.1 \\
(70.8- \\
71.4)\end{array}$ & $\begin{array}{c}70.3 \\
(69.0- \\
71.6)\end{array}$ & 0.2 & $\begin{array}{c}64.4 \\
(63.8- \\
65.1)\end{array}$ & $\begin{array}{c}76 \\
(75.5- \\
76.6)\end{array}$ & $<0.001$ & $\begin{array}{c}69.6 \\
(69.4- \\
70.0)\end{array}$ & $\begin{array}{c}74.5 \\
(74.1-75)\end{array}$ & $<0.001$ \\
\hline $\begin{array}{l}\text { Producción } \\
\text { de embriones }\end{array}$ & $\begin{array}{l}20.7 \\
(20.5- \\
21.0)\end{array}$ & $\begin{array}{c}22.2 \\
(21.0- \\
23.2)\end{array}$ & 0.01 & $\begin{array}{c}21.5 \\
(21-22.1)\end{array}$ & $\begin{array}{l}28.2 \\
(27.7- \\
28.8)\end{array}$ & $<0.001$ & $\begin{array}{c}20.8 \\
(20.6- \\
21.2)\end{array}$ & $\begin{array}{l}26.5 \\
(26.0- \\
27.0)\end{array}$ & $<0.001$ \\
\hline $\begin{array}{l}\text { Preñeces } \\
\text { obtenidas }\end{array}$ & $\begin{array}{c}44.4 \\
(42.0- \\
46.1)\end{array}$ & $\begin{array}{c}49.4 \\
(42.6- \\
56.4)\end{array}$ & 0.1 & $\begin{array}{c}45.1 \\
(42.4- \\
47.9)\end{array}$ & $\begin{array}{c}46 \\
(43.7- \\
48.4)\end{array}$ & 0.62 & $\begin{array}{c}44.6 \\
(43.2- \\
46)\end{array}$ & $\begin{array}{c}46.0 \\
(43.8- \\
48.2)\end{array}$ & 0.3 \\
\hline
\end{tabular}

Intervalo de confidencia al 95\% entre paréntesis

lidad del oocito, aspecto que no se evaluó en este estudio. Mejía (2010) reportó que la movilidad de los espermatozoides en el semen de toros Bos indicus se afecta menos que el semen de toros Bos taurus por el proceso de sexaje.

Si bien se describe que la técnica de sexaje del semen afecta la competencia espermática y la habilidad de desarrollo embrionario al alterar componentes de la membrana celular (Inaba et al., 2016), algunos trabajos reportan que no existe una diferencia en las tasas de desarrollo de blastocisto usando semen sexado (Lu y Seidel, 2004; Zhang et al., 2003; Carvalho et al., 2010).

Blondin et al. (2009) obtuvieron mayor división embrionaria, pero menores tasas de blastocisto usando semen sexado. Las diferencias pueden atribuirse a cambios en los protocolos, al aumentar la concentración de espermatozoides (Barceló-Fimbres et al.,
2011), y al aplicar estrategias para reducir el daño del sexado (Rath et al., 2013). Por otro lado, Contreras et al. (2015) reportaron tasas de división embrionaria similares entre semen convencional y sexado; sin embargo, el uso de semen sexado disminuyó la formación de blastocisto $(15.6 \%$ vs. $18.9 \%$, respectivamente).

Tanno (2009) y Mejía (2010) afirman que el semen sexado de Bos indicus tiene mayor resistencia para soportar los daños de la integridad de la membrana plasmática, pero se desconoce el mecanismo que lo hace más resistente. No obstante, algunos trabajos reportan resultados similares al obtenido en este estudio sobre la tasa de división embrionaria, donde hubo una disminución en el desarrollo de la tasa de blastocisto al usar semen sexado (Merton et al., 1997; Bermejo-Alvarez et al., 2008; Palma et al., 2008; Stinshoff et al., 2012). 
Carvalho et al. (2010) concluyeron que el procedimiento de sexado por citometría de flujo afectó a algunas características estructurales de los espermatozoides bovinos, pero no redujo su capacidad para producir embriones in vitro. Además, los embriones masculinos y femeninos producidos por espermatozoides clasificados tuvieron un desarrollo similar in vitro.

En el presente trabajo no hubo diferencia significativa en ninguna de las comparaciones sobre la tasa de preñez, obteniéndose una tasa de preñez general de $46 \%$, siendo de $44.4 \%$ con semen sexado. Pellegrino et al. (2016) obtuvo tasas de preñez de $35.4 \%$ con semen sexado, en tanto que Pontes et al. (2011) obtuvieron $36 \%$ de preñez con semen sexado. Asimismo, Pontes et al. (2010) reportan tasas de preñes de $40 \%$ para vacas Gyr, 36\% para vacas Holstein, $37 \%$ para $1 / 4$ Holstein 3/4 Gyr, y 37\% para 1/2 HolsteinGyr utilizando semen sexado. Xu et al. (2009) por otro lado, reportaron tasas de preñez de $40 \%$ y $\sin$ diferencia significativa entre el uso de semen sexado o semen convencional para la producción de embriones in vitro.

\section{ConClusiones}

- La producción de embriones y la tasa de división embrionaria fue mejor con el semen convencional en comparación con el semen sexado.

- No hubo diferencia significativa en la utilización de semen convencional o semen sexado en la tasa de preñez de animales Bos taurus y Bos indicus.

\section{Literatura Citada}

1. Bavister BD. 1989. A consistently successful procedure for in vitro fertilization of golden hamster eggs. Gamete Res 23: 139-158. doi: 10.1002/ mrd.1120230202
2. Barceló-Fimbres M, Campos-Chillón LF, Seidel GE. 2011. In vitro fertilization using non-sexed and sexed bovine sperm: sperm concentration, sorter pressure, and bull effects. Reprod Domest Anim 46: 495-502. doi: 10.1111/ j.1439-0531.2010.01696.x

3. Bermejo-Alvarez P, Rizos D, Rath D, Lonergan P, Gutiérrez-Adán A. 2008. Can bovine in vitro-matured oocytes selectively process X- or Y-sorted sperm differentially? Biol Reprod 79: 594-597. doi: 10.1095/biolreprod.108.070169

4. Blondin P, Beaulieu M, Fournier V, Morin N, Crawford L, Madan P, King $W A$. 2009. Analysis of bovine sexed sperm for IVF from sorting to the embryo. Theriogenology 71: 30-38. doi: 10.1016/j.theriogenology.2008.09.017

5. Carvalho JO, Sartori R, Machado GM, Mourão GB, Dode MA. 2010. Quality assessment of bovine cryopreserved sperm after sexing by flow cytometry and their use in vitro embryo production. Theriogenology 74: 1521-1530. doi: 10.1016/j.theriogenology.2010.06.030

6. Contreras WO, Alvis-Miranda HR, Gamarra AF, Rendon B, Borda DA, Albicker $U$, et al. 2015. Effects of sexed semen and interactive effects on commercial in vitro embryo production when oocytes are collected from cows of Bos indicus, and Bos taurus breeding and crossbred cows of these subspecies. Anim Reprod Sci 156: 58-63. doi: 10.1016/j.anireprosci.2015.02.009

7. Galli C, Lazzari G. 2008. The manipulation of gametes and embryos in farm animals. Reprod Domest Anim 43 (Suppl 2): 1-7. doi: 10.1111/j.14390531.2008.01136.x

8. Gordon I. 1994. Laboratory production of cattle embryos. Cambridge: CAB International. $640 \mathrm{p}$.

9. Guerreiro BM, Batista EO, Vieira LM, Sá Filho MF, Rodrigues CA, Castro $A$, et al. 2014. Plasma anti-Mullerian hormone: an endocrine marker for in vitro embryo production from Bos taurus 
and Bos indicus donors. Domest Anim Endocrin 49: 96-104. doi: 10.1016/ j.domaniend.2014.07.002

10. Hinde K, Carpenter AJ, Clay JS, Bradford BJ. 2014. Holsteins favor heifers, not bulls: biased milk production programmed during pregnancy as a function of fetal sex. Plos One 9: e86169. doi: 10.1371/journal.pone.0086169

11. Inaba Y, Abe R, Geshi M, Matoba S, Nagai T, Somfai T. 2016. Sex-sorting of spermatozoa affects developmental competence of in vitro fertilized oocytes in a bull-dependent manner. J Reprod Develop 62: 451-456. doi: 10.1262/ Jrd.2016-032

12. Jo HT, Bang J, Kim SS, Choi BH, Jin JI, Kim HL, Jung IS, et al. 2014. Production of female bovine embryos with sex-sorted sperm using intracytoplasmic sperm injection: Efficiency and in vitro developmental competence. Theriogenology 81: 675-682. doi: 10.1016/j.theriogenology.2013.11.010

13. Lonergan P, Fair T. 2008. In vitroproduced bovine embryos: dealing with the warts. Theriogenology 69: 17-22. doi: 10.1016/j.theriogenology.2007.09.007

14. Lu KH, Seidel GE. 2004. Effects of heparin and sperm concentration on cleavage and blastocyst development rates of bovine oocytes inseminated with flow cytometrically-sorted sperm. Theriogenology 62: 819-830. doi: 10.1016/j.theriogenology.2003.12.029

15. Mejía A. 2010. Avaliação das características da motilidade (CASA), morfologia e funcionalidade da membrana plasmática (HOST) de espermatozóides bovinos sexados por citometria de fluxo. Tesis de Maestría. Dao Paulo, Brasil: Universidade de São Paulo.

16. Merton JS, Haring RM, Stap J, Hoebe RA, Aten JA. 1997. Effect of flow cytometrically sorted frozen/thawed semen on success rate of in vitro bovine embryo production. Theriogenology 47: 295.

17. Morotti F, Sanches BV, Pontes JHF, Basso AC, Siqueira ER, Lisboa LA, Seneda MM. 2014. Pregnancy rate and birth rate of calves from a large-scale IVF program using reverse-sorted semen in Bos indicus, Bos indicus-taurus, and Bos taurus cattle. Theriogenology 81: 696-701. doi: 10.1016/j.theriogenology.2013.12.002

18. Nasser LF, Reis EL, Oliveira MA, Bó GA, Baruselli PS. 2004. Comparison of four synchronization protocols for fixed-time bovine embryo transfer in Bos indicus x Bos taurus recipients. Theriogenology 62: 1577-1584. doi: 10.1016/ j.theriogenology.2004.03.013

19. Palma GA, Olivier NS, Neumüller C, Sinowatz F. 2008. Effects of sex-sorted spermatozoa on the efficiency of in vitro fertilization and ultrastructure of in vitro produced bovine blastocysts. Anat Histol Embryol 37: 67-73. doi: 10.1111/j.14390264.2007.00795.x

20. Parrish JJ, Susko-Parrish JL, Leibfriedge-Ruthedge ML, Critser ES, Eyestone WH, First NL. 1986. Bovine in vitro fertilization with frozen thawed semen. Theriogenology 25: 591-600.

21. Parrish JJ, Susko-Parrish J, Winer MA, First NL. 1988. Capacitation of bovine sperm by heparin. Biol Reprod 38: 1171-1180.

22. Pellegrino CA, Morotti F, Untura RM, Pontes JH, Pellegrino MF, Campolina JP, et al. 2016. Use of sexed sorted semen for fixed-time artificial insemination or fixed-time embryo transfer of in vitro-produced embryos in cattle. Theriogenology 86: 888-893. doi: 10.1016/j.theriogenology.2016.03.010

23. Perez BC, Peixoto MG, Bruneli FT, Ramos PV, Balieiro JC. 2016. Genetic analysis of oocyte and embryo production traits in Guzerá breed donors and their associations with age at first calving. Genet Mol Res 15(2). doi: 10.4238/ gmr. 15027583

24. Plourde D, Vigneault C, Laflamme I, Blondin P, Robert C. 2012. Cellular and molecular characterization of the impact of laboratory setup on bovine in vitro embryo production. Theriogenology 77 : 1767-1778. doi: 10.1016/j.theriogenology.2011.12.021 
25. Pontes JH, Silva KC, Basso AC, Rigo $A G$, Ferreira CR, Santos GM, Sanches $B V$, et al. 2010. Large-scale in vitro embryo production and pregnancy rates from Bos taurus, Bos indicus, and indicus-taurus dairy cows using sexed sperm. Theriogenology 74: 1349-1355. doi: $10.1016 / j$.theriogenology.2010.06.004

26. Pontes JHF, Melo FA, Basso AC, Ferreira CR, Sanches BV, Rubin KCP, Seneda MM. 2011. Ovum pick up, in vitro embryo production, and pregnancy rates from a large-scale commercial program using Nelore cattle (Bos indicus) donors. Theriogenology 75 : 1640-1646. doi: 10.1016/j.theriogenology.2010.12.026

27. Rasmussen S, Block J, Seidel GE, Brink $Z$, McSweeney $K$, Farin $P W$, Bonilla L, et al. 2013. Pregnancy rates of lactating cows after transfer of in vitro produced embryos using $\mathrm{X}$-sorted sperm. Theriogenology 79: 453-461. doi: 10.1016/j.theriogenology.2012.10.017

28. Rath D, Barcikowski S, de Graaf S, Garrels W, Grossfeld R, Klein S, Knabe W, et al. 2013. Sex selection of sperm in farm animals: status report and developmental prospects. Reproduction 145: 15-30. doi: 10.1530/REP-12-0151

29. Seneda MM, Esper CR, Garcia JM, Oliveira JA, Vantini R. 2001. Relationship between follicle size and ultrasound-guided transvaginal recovery. Anim Reprod Sci 67: 37-43.

30. Smith LC, Olivera-Angel M, Groome NP, Bhatia B, Price CA. 1996. Oocyte quality in small antral follicles in the presence or absence of a large dominant follicle in cattle. J Reprod Fertil 106: 193199.
31. Stinshoff H, Krienke M, EkhlasiHundrieser M, Wilkening S, Hanstedt A, Frese D, Rath D, et al. 2012. Seminal plasma and seminal plasma proteins added to bulk sorted sperm do not alter the mRNA expression of in vitro produced bovine embryos. Theriogeno$\log$ 78: 132-139. doi: 10.1016/j.theriogenology.2012.01.028

32. Sugimura S, Akai T, Imai K. 2017. Selection of viable in vitro-fertilized bovine embryos using time-lapse monitoring in microwell culture dishes. J Reprod Develop 63: 353-357. doi: 10.1262/jrd.2017-041

33. Tanno PH. 2009. Estudo das alterações morfo-funcionais de espermatozóides bovinos submetidos à sexagem por meio da técnica de citometria de fluxo. Tesis de Maestría. Sao Paulo, Brasil: Universidade de São Paulo. 100 p.

34. Viana JH, Siqueira LG, Palhao MP, Camargo LSA. 2012. Features and perspectives of the Brazilian in vitro embryo industry. Anim Reprod 9: 12-18.

35. Wright J. 1998. Photomicrographic illustration of embryo codes. In: Stringfellow DA, Seidel SM (eds). Manual of the international embryo transfer society. $3^{\text {rd }}$ ed. Savory, IL: IETS. p167170.

36. Xu J, Chaubal SA, Du F. 2009. Optimizing IVF with sexed sperm in cattle. Theriogenology 71: 39-47. doi: 10.1016/j.theriogenology.2008.09.012

37. Zhang M, Lu KH, Seidel GE. 2003. Development of bovine embryos after in vitro fertilization of oocytes with flow cytometrically sorted, stained and unsorted sperm from different bulls. Theriogenology 60: 1657-1663. doi: 10.1016/S0093-691X(03)00177-8 